\title{
Sonar and its Use in Kidney Disease in Children
}

\author{
E. A. LYONS, ^ A. V. MURPHY, and G. C. ARNEIL \\ From the Department of Ultrasonics, Queen Mother's Hospital; Department of Child Health and Renal Unit, Roval \\ Hospital for Sick Children, Glasgow
}

\begin{abstract}
Lyons, E. A., Murphy, A. V., and Arneil, G. C. (1972). Archives of Disease in Childhood, 47, 777. Sonar and its use in kidney disease in children. The basic principles of diagnostic ultrasound or sonar are given, together with the special technique required for scanning newborn infants and small children for kidney abnormalities.

Illustrative examples of the potential of this procedure, both in diagnosis and in monitoring changes include a normal neonatal and preadolescent kidney, unilateral renal agenesis, duplex kidney, renal cyst, polycystic disease, nephroblastoma, and examples of mild and severe hydronephrosis.
\end{abstract}

Sonar (pulsed ultrasound) is a non-invasive method of investigation used to delineate the gross anatomy of structures irrespective of their function. Its potential in the investigation and monitoring of renal disorders in adults has been known since 1961 (Schlegel, Diggdon, and Cuellar), but only very recently has it been used in older children (Hünig and Ameri, 1970). We here outline the basic principles of ultrasound, and illustrate its application in establishing the final diagnosis in kidney disease in infants and young children.

\section{History}

Ultrasound waves with a frequency beyond the range of human hearing (i.e. 20,000 cycles per second, or $20,000 \mathrm{Hertz}$ ) were first produced in 1880 by Curie and Curie. They noted that a quartz crystal would vibrate when it was subjected to an electrical charge. Conversely, a voltage was produced when these ultrasound waves caused a quiescent crystal to vibrate. This ability to convert electrical to mechanical energy was called the piezoelectric effect (Langevin, 1928) found in many natural crystals as well as in some synthetic ceramics. The latter are commonly in use today. Two important features of this system allowed it to develop. Firstly, by varying the thickness of the crystal one could vary the characteristic frequency at which it would vibrate, and therefore its properties of penetration and resolution. Secondly, the

\footnotetext{
Received 21 June 1972.

*On secondment from the Winnipeg General Hospital, Manitoba, Canada.
}

crystal could be so encased as to provide a unidirectional beam which would reflect off objects with physical properties differing from those of the surrounding medium. These reflected waves, or 'echoes', could then be detected by the same crystal and the position of the object recorded relative to it. The first ultrasonic generator was made in 1917 in France for the detection of large underwater objects (U-boats!). Ten years later there was a successful attempt to localize intracerebral masses through the intact skull.

\section{Principles of Ultrasonic Scanner}

The composition of a basic ultrasonic system may be conveniently divided into three major parts: the pulse generator, the transducer, and the signal processor (Fig. 1 ( $i$ and ii)).

Pulse generator. This is the source of the high voltage electrical charge used to excite the transducer. The number of excitation pulses or the pulse repetition rate varies from 300 to 1000 per second. This section has a dual function in that it also generates the horizontal baseline or ' $\mathrm{X}$-axis' on the oscilloscope screen.

Transducer. The flat or slightly focused piezoelectric crystal emits a highly directional beam of very high frequency sound waves in response to an electrical stimulus from the pulse-generator. This ultrasonic beam will not penetrate air; therefore, between the transducer and the skin surface there must always be an ample amount of contact medium, such as olive oil, peanut oil, or liquid paraffin. This effect of air also limits the present use of ultrasound to the investigation of solid or fluid containing organs or cavities.

In the body, as the beam strikes an interface of two 


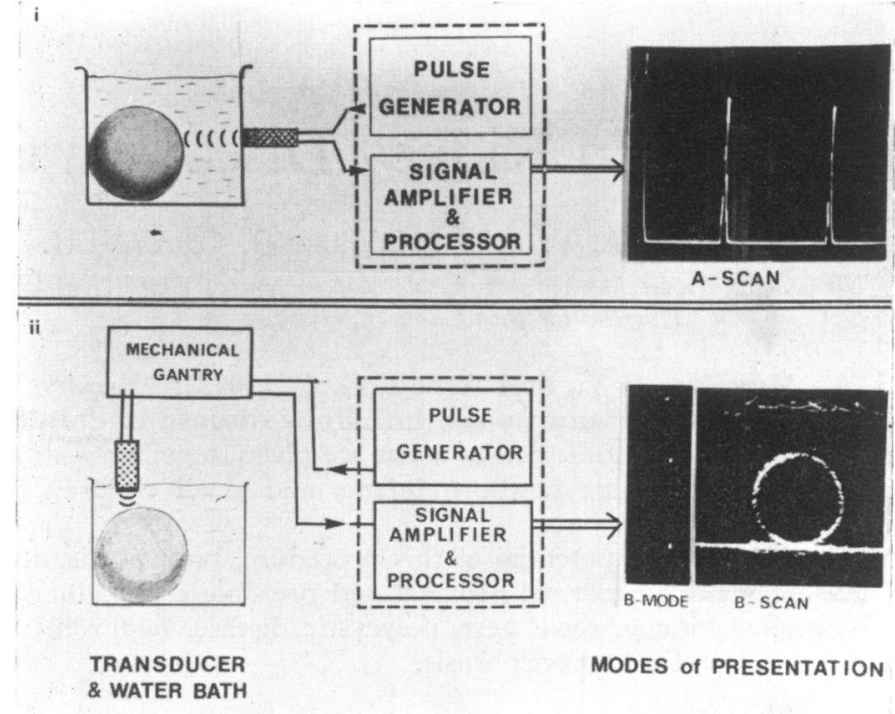

FIG. 1.-Diagrammatic representation of an ultrasonic scanner. Above (i) is shown the principal components of an $A$-scan system and below (ii) those of a B-mode and B-scan system.

substances of differing physical properties, it will be partly transmitted, partly refracted, and partly reflected, depending on the angle of incidence. When the long axis of the beam is perpendicular to an interface a large proportion will be reflected back to the emitting crystal causing it to vibrate. This in turn creates an electrical current which is passed on to the signal amplifier and processor.

Signal processor. At this stage the multitude of echoes varying in shape and in intensity are modified, selectively amplified, and finally presented on an oscilloscope in any one of three modes.

$A$-scan (Fig. 1 (i)). The interfaces are represented as vertical deflections on a baseline which remains horizontal, regardless of its spatial relation to the transducer. The distance between any two deflections can be accurately measured by means of electronic calipers or by superimposing a grid of known dimensions. The height of an unprocessed echo depends on the physical characteristics of the interface from which the echo arose, and the amount of attentuation of sound in the tissue through which the beam has passed.

B-scan (Fig. 1 (ii)). As opposed to A-scan, in this mode of presentation the baseline no longer remains horizontal but now bears a spatial relation to the long axis of the transducer (i.e. when the transducer is vertical in position, the baseline on the oscilloscope will also be vertical). This effect is achieved through a system of three potentiometers housed in the mechanical gantry. The echoes, seen as vertical deflections on A-scan, are converted to dots of light, whose intensity varies with the echo strength. This is called B-mode.
As the transducer is passed over an object or a section of the body, echoes will be produced from all major interfaces in that one plane and will be retained on a storage oscilloscope as a cross-sectional image of the area scanned. This is a B-scan and is rather like a tomographic cut.

All ultrasonic scans of the kidneys are carried out with the patient in the prone position, unless otherwise indicated. Longitudinal scans are designated with an $\mathrm{L}$ and are viewed as sections seen from the patient's left side, while transverse scans are designated with a $T$ and are displayed as though the observer were standing at the patient's feet.

The resolution of the system is influenced by the frequency of the transducer crystal and, to a lesser extent, by the distance of the object from that crystal. As the frequency is increased one gets better resolution but less penetration. Therefore, high frequencies, i.e. that of $5 \times 10^{6}$ cycles per second or 5 Megahertz $(\mathrm{MHz})$, are used to investigate relatively superficial structures, such as the eyes. Visualization of the infant kidneys may be somewhat improved at $5 \mathrm{MHz}$; however $2.5 \mathrm{MHz}$ is usually adequate. The $1.5 \mathrm{MHz}$ crystal provides information in areas difficult to penetrate, such as the skull.

For examinations requiring fine details it is inadvisable to place the object of interest too near the transducer face. The 'near zone', that is the first 2 to $4 \mathrm{~cm}$ of the beam, is an area of definitive nonuniformity and, therefore, in most scanners, information from it is electronically reduced. In adults this zone contains only subcutaneous tissue and muscles, whereas in infants it encompasses many vital organs. In an effort 


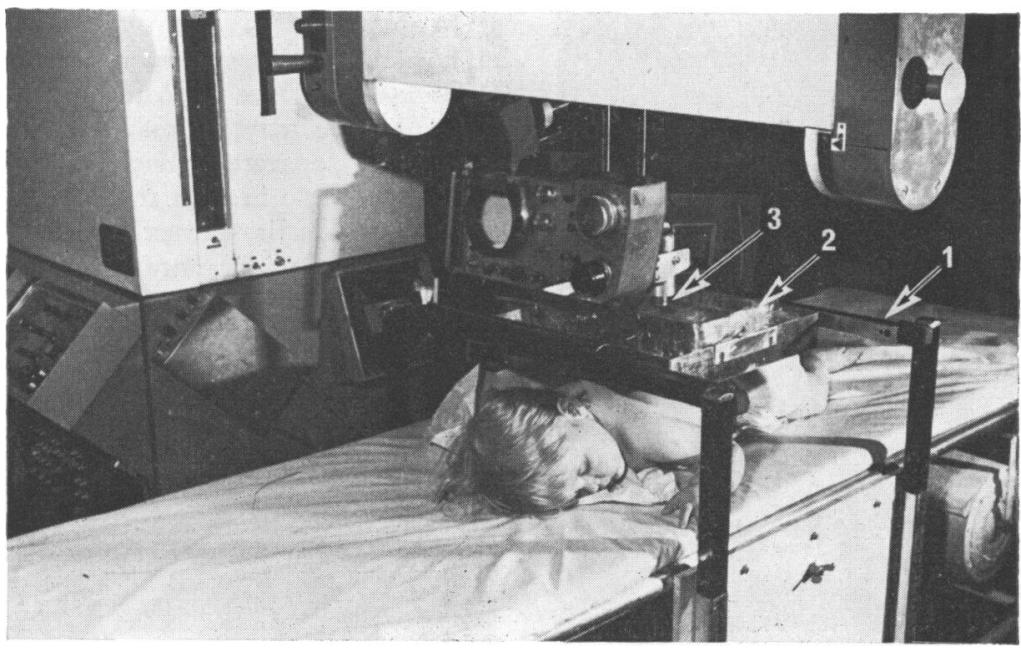

to avoid this zone we have designed a waterbath which serves to separate the transducer from the patient. Its physical structure has been described in our previous publication (Lyons et al., 1972). Previously children under the age of about 18 months could not usually be examined by the regular method of contact scanning. The waterbath technique for this group served first to offset the transducer, thereby avoiding the near zone effect and also maintain good transducer-patient contact throughout. Secondly, it kept the child warm and relatively still. When combined with mild sedation, sleep usually ensued. In the older child the kidneys are larger and the patient more co-operative, and standard contact scanning becomes possible.

Technique. The sedated child is placed prone on the examining trolley and the water bag is assembled as in Fig. 2. Olive oil is liberally applied to the renal area and all the visible air is excluded from between the polythene bag and the skin. With the transducer face wholly immersed in the water, longitudinal and transverse scans of both kidneys are obtained, noting specifically the size and shape of the kidney and its collecting system. The ureters, if grossly dilated, can be visualized on a transverse scan just cephalad to the iliac crests.

The normal bladder is best examined with the patient in the supine position. However, if it is abnormally distended and rises out of the pelvis, it can be seen even when the patient is prone.

The examination at present takes 30 to 45 minutes, but undoubtedly this time will be reduced with further experience.

\section{Results}

Normal kidney. B-scan is employed to show the position and configuration of the kidney.
Fig. 3 shows the appearance of normal kidneys in a newborn child. Fig. 4 shows normal kidneys in a pubescent child. The kidney of the older child as shown on longitudinal scanning $(L)$ is seen to be more elongated than that of the baby, but on transverse scanning $(T)$ the ovoid configuration is maintained in both. The pelvicalyceal system appears as a centrally located uniform collection of echoes, save the exception that on transverse section at the level of the hilum it is eccentric and normally lies on the medial renal border. The transverse scan ( $T$ ) in Fig. 3 shows that on a single section one kidney may appear larger than the other. This can be due to the fact that the right kidney is situated lower than the left and the level of maximum circumference is therefore different for each. The adrenal gland can be clearly seen as a rounded echo-free area at the upper pole of each kidney. In the child the left adrenal is outlined more readily than the right, whereas in adults neither is usually well seen. Demonstration of the actual physical location of the kidney is a help to accurate acupuncture in the form of percutaneous renal biopsy. Demonstration of the existence of a kidney is also of importance in situations such as renal venous thrombosis. The existence of haematuria, thrombocytopenia, and nitrogen retention in a young infant makes this a likely diagnosis. In only half of these cases is an enlarged kidney felt. If an intravenous pyelogram shows no function on one (or both) side then B-scanning will reveal whether or not there is a kidney present. This is a useful complement to 

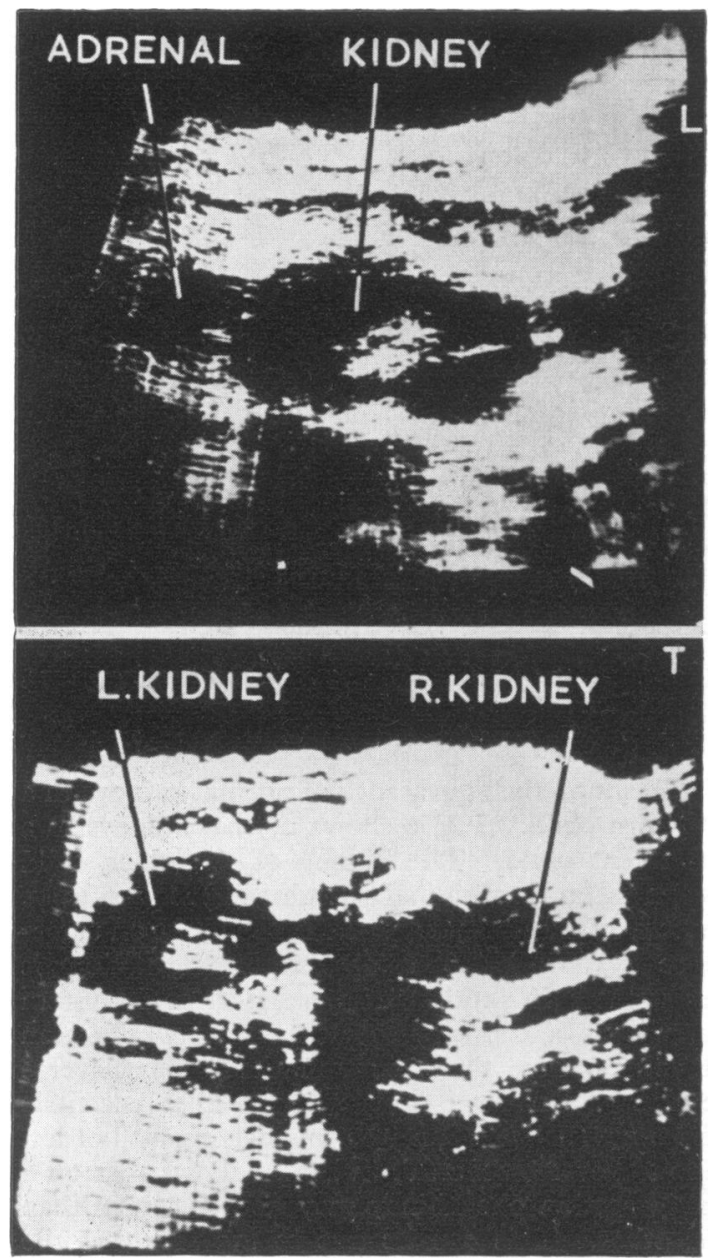

FIG. 3.-Above a longitudinal ( $L$ ) scan and below a transverse $(T)$ scan of a kidney in a normal neonate. The scan was made using the water bath technique.

the data obtained from tomography which may be inconclusive.

Renal agenesis. The newborn with the classical 'Potter' facies, anuria, and progressive uraemia makes diagnosis relatively simple, but not all cases are so obvious. In Fig. 5 is a composite of three B-scans of a 12-year-old boy found to have unilateral nonfunction of an intravenous pyelogram. The transverse section ( $T$ ) shows an enlarged right kidney, but no kidney on the left. The diagnosis of renal agenesis was confirmed by a midline scan of the pelvis to exclude an ectopic or pelvic kidney.
Duplex kidney. On ultrasonography the duplex kidney appears as larger than normal and with a definite interface dividing it in two (Fig. 6). This interface may, or may not, be easily demonstrable. A separate pelvicalyceal complex should be demonstrable in each moiety except when gross hydronephrosis is present. The upper part of the duplex kidney is more liable to hydronephrosis which may appear initially as an irregular cystic structure closely applied to a foreshortened kidney.

Solitary renal cyst. A nonfunctioning renal
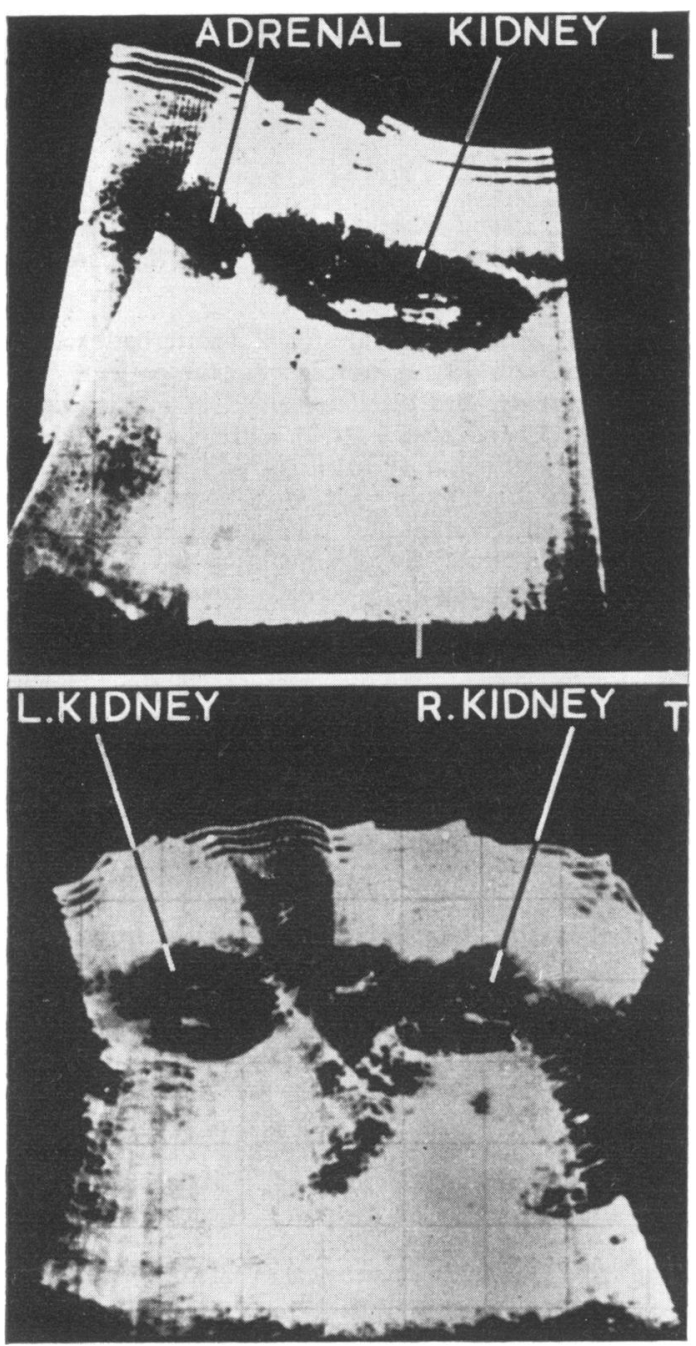

FIG. 4.- Above longitudinal ( $L$ ) scan and below transverse $(T)$ scan of a kidney in a normal 12-year-old girl, using direct contact scanning. 

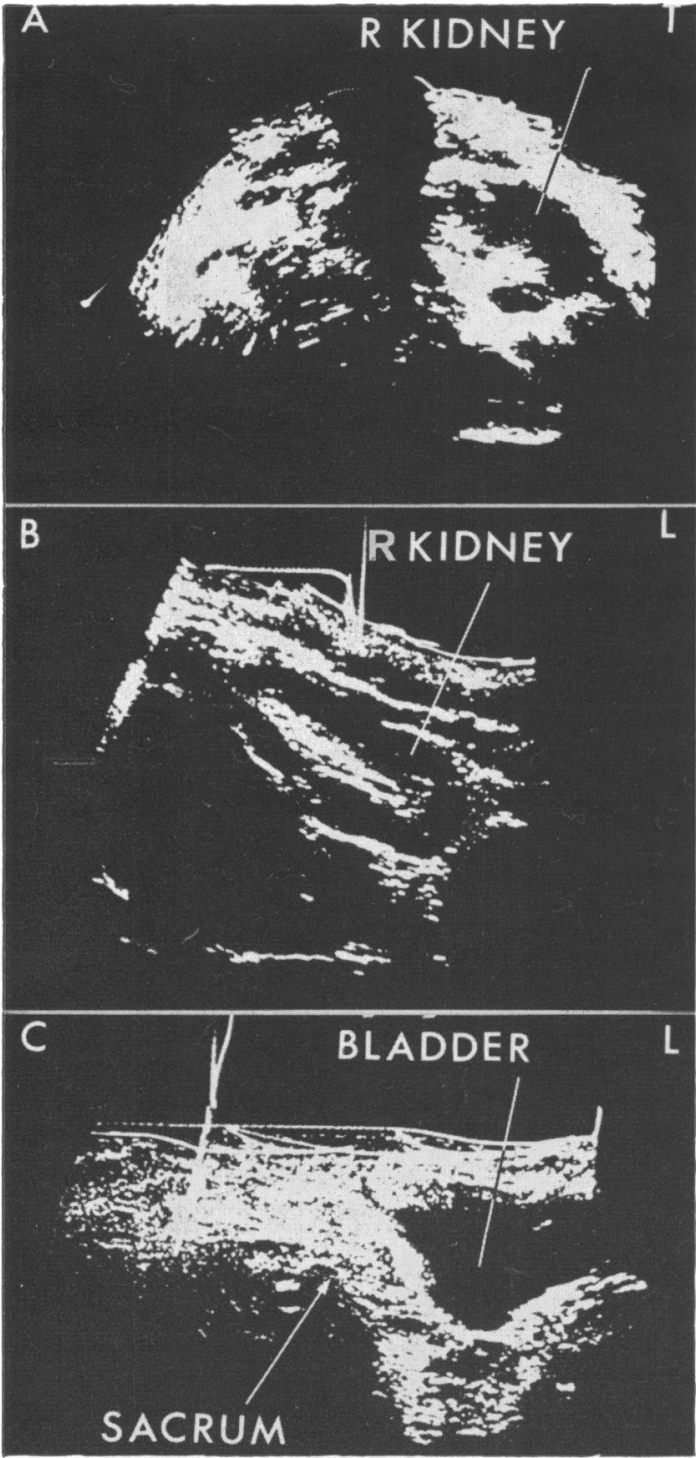

Fig. 5.-(A) Transverse scan at the level of the midposition of the right kidney in a patient with unilateral renal agenesis. (B) Longitudinal scan of the enlarged solitary kidney. (C) Longitudinal midline scan of the pelvis and distended bladder, in the supine position.

area noted on intravenous pyelogram may suggest the possibility of a renal cyst, as happened in the child shown in Fig. 7. The ultrasonic scan (longitudinally) shows a cyst with a sharply defined periphery and an echo-free centre. Structurally, a cyst distorts renal architecture (in this example, the upper pole) and thus differs from the relatively echo-free adrenal gland usually seen in this position. In this child a scan of the other kidney revealed a previously unsuspected cyst.

The ability to differentiate between a cystic and a solid mass is one of the main advantages of ultrasound. Fig. 8 illustrates how this is done. At low levels of equipment sensitivity a cyst and a tumour will appear similar on B-scan. At high sensitivity the cyst will remain echo free on B-scan and A-scan, whereas a solid tumour will be filled with echoes arising from its many microscopical and macroscopical tissue interfaces.

Polycystic disease of kidneys. When a family history suggests the possibility that a child may be affected with adult type polycystic disease, repeated intravenous pyelography may not be desirable and ultrasonic scanning offers an alternative form of monitoring the disease. The tracing in Fig. 9 was obtained from an 8-year-old girl with a positive family history and a suggestive intravenous pyelogram. On this ultrasound tracing there is gross distortion of the calyceal echo pattern and many small cysts can be seen as curvilinear lines around an echo-free area. Cysts of less than 1.5 $\mathrm{cm}$ diameter are undetectable with the existing equipment and consequently the early case will present with a normal ultrasonogram which will later change.

Nephroblastoma. The early nephroblastoma encapsulated and contained within the intact renal capsule is characteristically found accidentally either during laparotomy, as an unexpected distortion of an intravenous pyelogram, or when unexplained hypertension is investigated. Usually when the renal capsule ruptures the hypertension, which may be gross, disappears. In Fig. 10 are shown the ultrasonic findings in a child with nephroblastoma. When the boundaries of tumour are well defined it is usually encapsulated, but as local spread occurs the limits are less distinct. At high equipment sensitivities areas of mucoid degeneration appear in the tumour as irregular echo-free spaces within an otherwise solid mass.

Hydronephrosis. Hydronephrosis is easily shown both in its mild and severe forms. In the example shown in Fig. 11 the pelvicalyceal system appears not as a normal solid white line but rather as a double line of echoes separated by a clear area. This is due to minimal distension of the system with urine and may be seen transiently during diuresis or constantly as in mild hydronephrosis. In both instances the kidney may be normal in size and shape. 

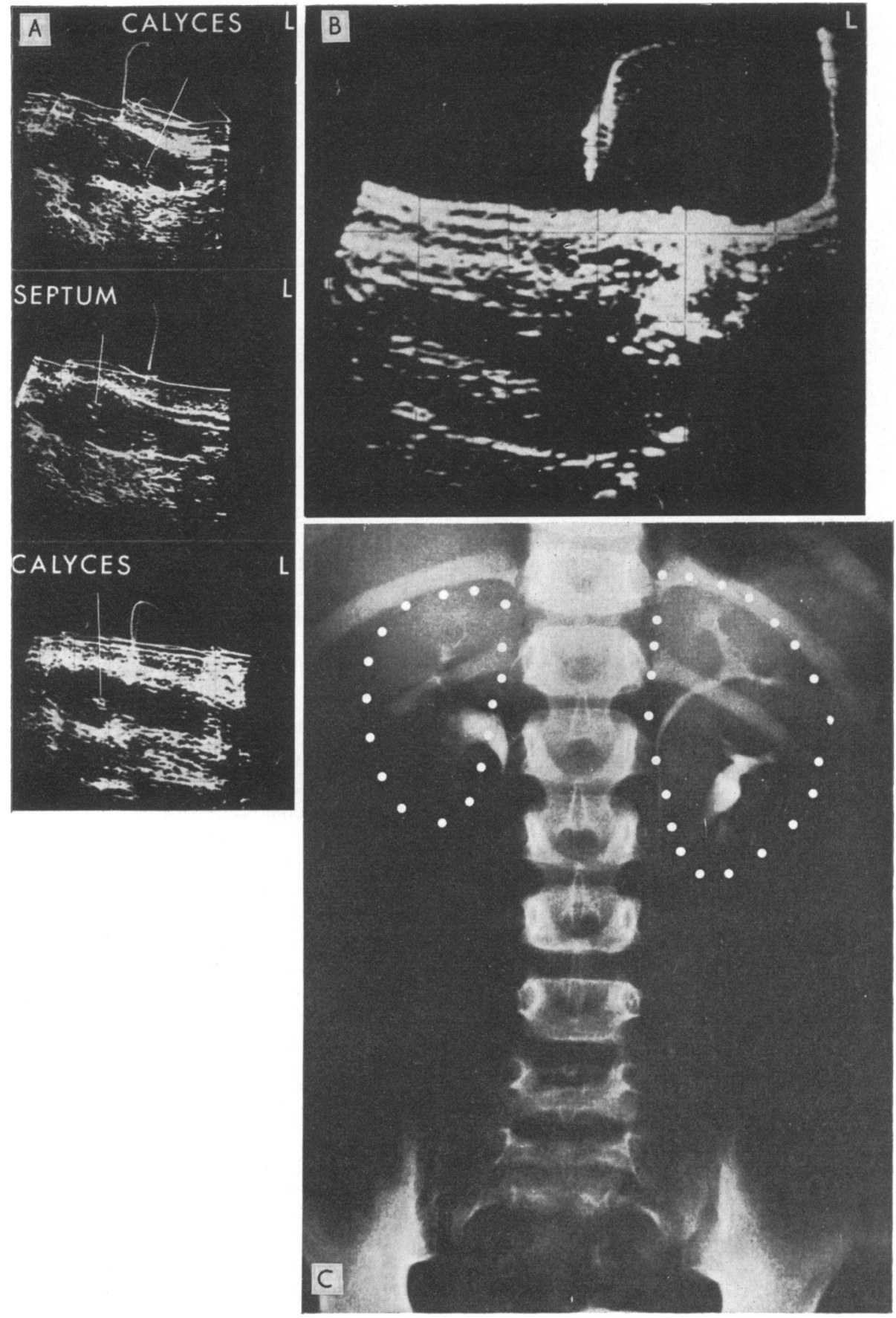

Fig. 6.-(A) Three longitudinal sections of a functioning duplex kidney, with the most lateral scan on top and the most medial on the bottom. (B) Longitudinal scan of the normal kidney. (C) Single film from the intravenous pyelogram obtained the day before the ultrasonic examination. 


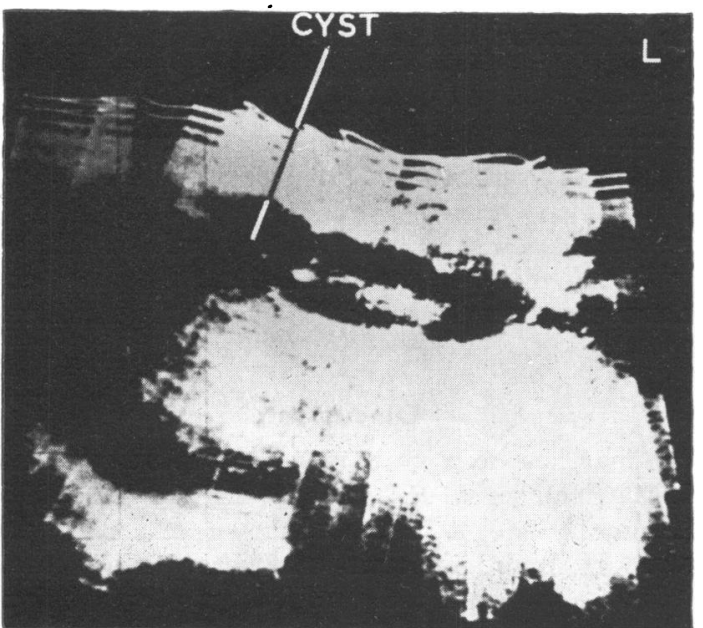

FIG. 7.-Longitudinal section of a kidney with a single cyst in the upper pole.

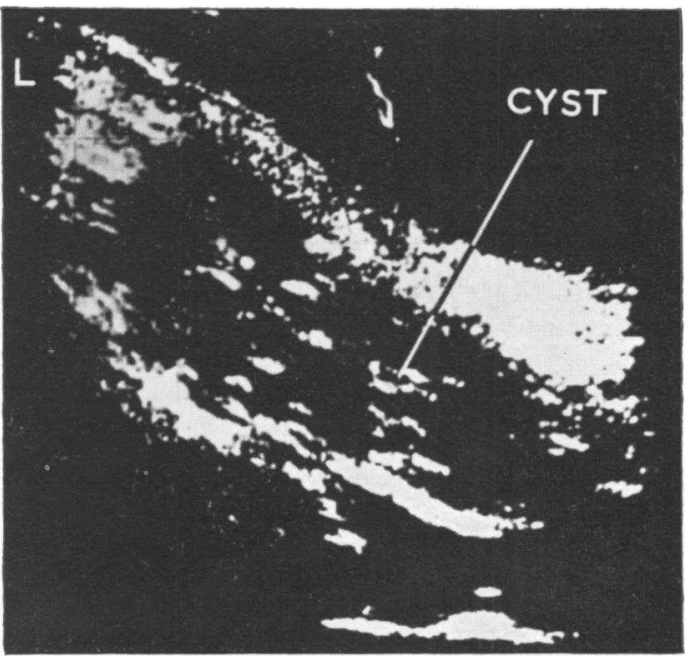

FIG. 9.-Longitudinal section of a polycystic kidney.

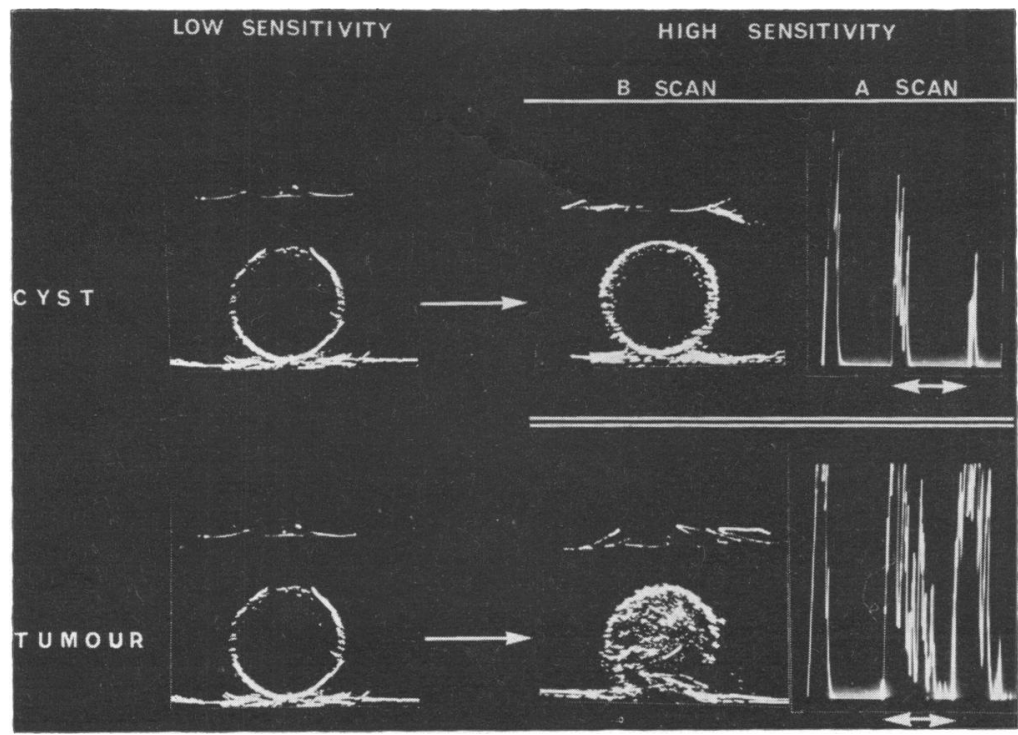

FIG. 8.-Ultrasound scans of a cyst and a solid structure tumour at low and high levels of equipment sensitivity. On A-scan the extent of the mass is indicated by $(\leftrightarrow \rightarrow)$. Echoes from the deep layers of the tumour are less prominent than those more proximal. This is due to the attenuation of sound by the tumour tissue. 


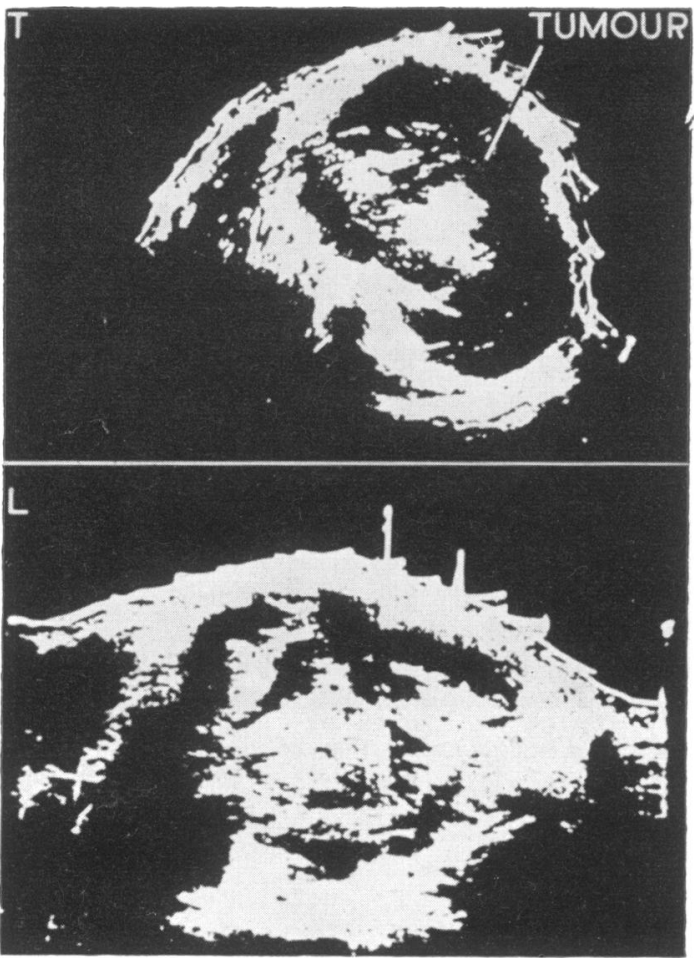

FIG. 10.-Above a transverse section $(T)$ and below a longitudinal $(L)$ section of a supine patient with massive a nephroblastoma replacing the left kidney.

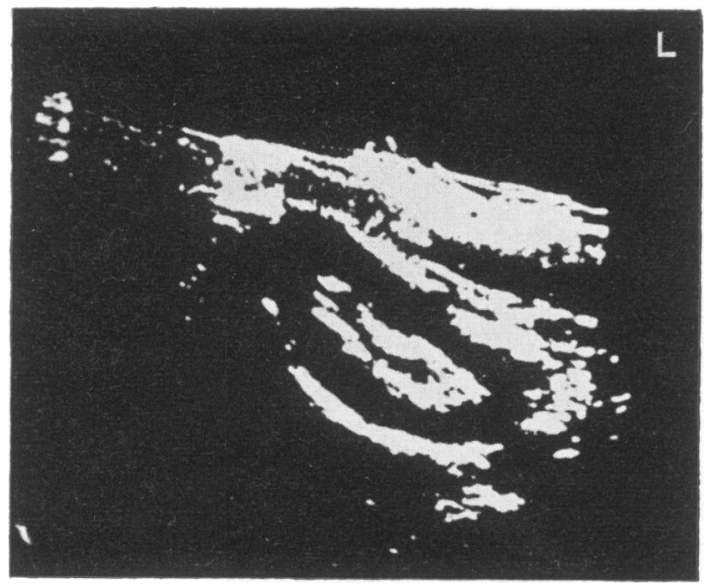

FIG. 11.-A longitudinal section of the kidney showing a mild degree of pelvicalyceal dilatation.

Megacystis-megaureter syndrome. In severe hydronephrosis the kidneys may be so distorted that ultrasonically they appear only as irregular echo-free spaces in the renal area. Often no definite cortex can be outlined (Fig. 12).

In the megacystis-megaureter syndrome the hydronephrosis is associated with grossly distended ureters and bladder. The ureters were shown by a transverse scan, in the prone position, at the level of the iliac crests. They appear as oblong, cystic structures closely applied to the posterior abdominal wall. In this case the bladder fills the pelvis and extends up to and beyond the level of the umbilicus.

\section{Discussion}

Sonar has been used in adult nephrology since 1961 (Schlegel et al.), and extensively since 1966 (Holmes, 1966). In the diagnosis of renal cysts, tumours, hydronephrosis, and perinephric abscess it is an established and useful diagnostic technique (Barnett and Morley, 1971). In children progress has been slower (Hünig and Ameri, 1970), and in infants impossible until now. This was especially unfortunate since such complemental aids to clarification of doubtful intravenous pyelography, such as nephrotomography, retrograde pyelography and angiography, not only may require an anaesthetic but in the very young child, are technically difficult, if not impossible.

In the investigation of paediatric or even neonatal renal disease we have shown that ultrasound can be a safe, nonsurgical, and nonionizing adjunct to the present armamentarium of the paediatric nephrologist. To ascertain its safety, a multicentric study was undertaken, involving 3297 pregnant women who had undergone one or more ultrasonic examinations during their pregnancy (Hellman et al., 1970). The degree of risk to the fetus was estimated by determining the frequency of congenital anomalies in the offspring of these patients. These authors concluded that, 'neither the frequency of ultrasound examination nor the time of the first examination seem to affect adversely the incidence of fetal abnormality and abortion; nor are abnormalities any more common than in the general population'.

From the available clinical data, therefore, this form of investigation appears to be safe for use in the paediatric age group. To the present date, experimental data from animal tissues and studies of human chromosomes have indicated that the power levels necessary to cause any significant damage are about 100 times that utilized in present diagnostic equipment (Watts, Hall, and Fleming, 1972).

When first applying new methods of investigation 

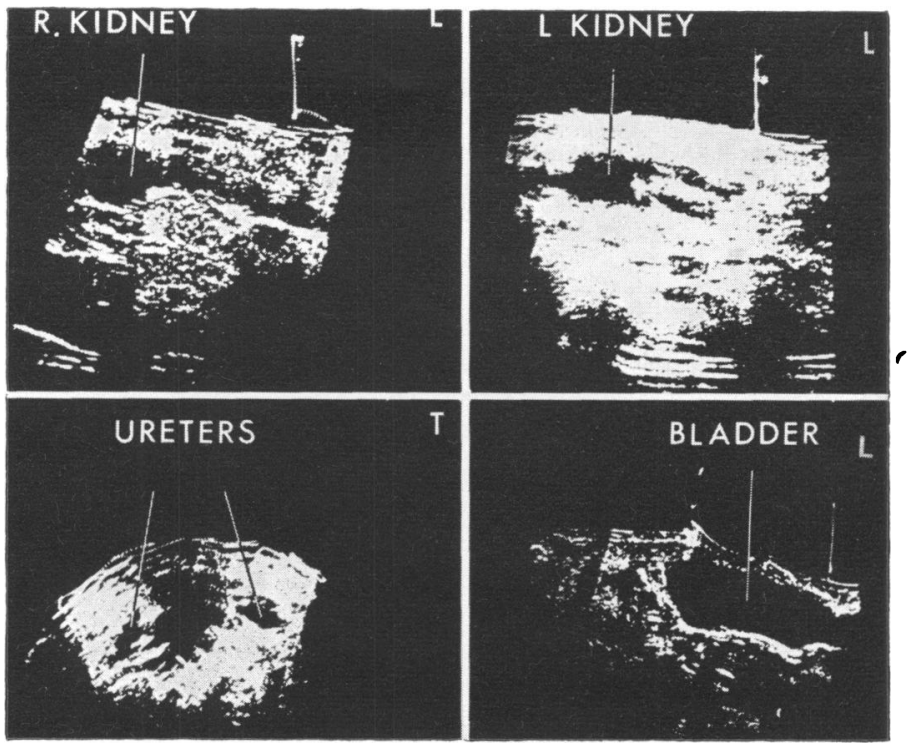

FIG. 12.-Above, longitudinal $(L)$ sections of the right and left renal areas in a patient with the megacystismegaureter syndrome. Below, transverse $(T)$ section just above the intercrestal line showing the dilated ureters and a midline longitudinal section in a supine position showing the grossly distended bladder.

to clinical problems it is easy to be carried away by enthusiasm and difficult to be objective. This is particularly so when faced with the spell-binding image of a B-scan building up on a persisting image oscilloscope a picture of what lies deep within a patient. From our brief experience to date it is clearly an important complementary form of investigation for a variety of renal conditions from birth to puberty. These uses include the following. (1) The localization and study of nonfunctioning kidney(s). (2) Distinguishing cystic from solid space-occuping lesions within the kidney. (3) The investigation of cystic and polycystic kidneys. (4) Demonstration of hydronephrosis and monitoring of subsequent progression (or improvement) with or without treatment. (5) Accurate localization of the kidney for percutaneous biopsy without use of ionizing radiation. (6) Where sensitivity to iodine inhibits intravenous pyelography.

Of these uses and others, which no doubt will evolve with time, one of the most important is likely to be in determining whether a nonfunctioning kidney in fact exists, and if so, where. In renal venous thrombosis this problem is commonly troublesome. Nonfunctioning gross hydronephrosis is easily shown by nephrosonography as is a renal tumour. The other attractive use is in distinguishing between cystic and solid masses in the renal parenchyma. Renal tumours are particularly well investigated by this atraumatic procedure.

In brief, ultrasonography is best used as a complement to radiology, and in this capacity promises to add much to our knowledge of paediatric renal disease.

We would like to acknowledge Professor Ian Donald, Regius Professor of Midwifery, for his help in this study; our radiologist colleagues Drs. E. M. Sweet, S. P. Rawson, and M. M. McNair; Mr. G. Donald, Department of Medical Illustration, Western Infirmary, Glasgow; and the M.R.C. for the provision of funds for technical aspects of the study.

\section{REFERENCES}

Barnett, E., and Morley, P. (1971). Ultrasound in the investigation of space-occupying lesions of the urinary tract. British fournal of Radiology, 44, 733.

Curie, J., and Curie, P. (1880). Sur l'électricité polaire dans les cristaux hémièdres à faces inclinée. Comptes Rendus de l'Academie des Sciences (Paris), 81, 383.

Hellman, L. M., Duffus, G. M., Donald, I., and Sundén, B. (1970). Safety of diagnostic ultrasound in obstetrics. Lancet, 1, 1133.

Holmes, J. H. (1966). Ultrasonic studies of the bladder and kidney. In Diagnostic Ultrasound-Proceedings of the First International Conference on Diagnostic Ultrasound, Pittsburgh, p. 465. Ed. by C. C. Grossman, G. H. Holmes, and C. Joyner. Plenum, London. 
Hünig, R., and Ameri, M. R. (1970). Abdominal ultrasound tomography in paediatrics. Fortschritte auf dem Gebiete der Rontgenstrahlen und der Nuklearmedizin, 113, 760.

Langevin, P. (1928). Les ondes ultrasonores. Revue Générale de l'Electricité, 23, 626.

Lyons, E. A., Fleming, J. E. E., Arneil, G. C., Murphy, A. V., Sweet, E. M., and Donald, I. (1972). Nephrosonography in infants and children-a new technique. British Medical fournal, 2, 689.
Schlegel, J. U., Diggdon, P., and Cuellar, J. (1961). The use of ultrasound for localizing renal calculi. Fournal of Urology, 86, 367.

Watts, P. L., Hall, A. J., and Fleming, J. E. E. (1972). Ultrasound and chromosome damage. British fournal of Radiology, 45, 335.

Correspondence to Professor G. C. Arneil, Department of Ultrasonics, The Queen Mother's Hospital, Yorkhill, Glasgow G3 85J. 